\title{
Tendencias de investigación en el aprendizaje ubicuo: un micro estudio de publicaciones seleccionadas del 2000 al 2015
}

\author{
Trends in Ubiquitous Learning Research: A Micro Study of Selected Publications \\ from 2000 to 2015
}

Mayela Coto-Chotto

mcoto@una.cr

Universidad Nacional de Costa Rica

Heredia, Costa Rica

Carmen Cordero-Esquivel

ccordero@una.cr

Universidad Nacional de Costa Rica

Heredia, Costa Rica

Sonia Mora-Rivera

smora@una.cr

Universidad Nacional de Costa Rica

Heredia, Costa Rica

Recibido-Received: 22/may/2016 / Corregido-Corrected: 4/ago /2016.

Aceptado-Accepted: 23/set/2016 / Publicado-Published: 31/jul /2017.

\begin{abstract}
Resumen
Este artículo analiza el alcance de la investigación en el aprendizaje ubicuo durante el período 20002015, para lo cual se revisaron artículos en inglés y en español que abordaran expresamente el tópico de "ubiquitous learning" o "aprendizaje ubicuo". Los artículos seleccionados se analizaron en las siguientes categorías: población meta, área temática abordada, tipo de contribución, país, medio de publicación y tipo de artículo. Los resultados indican que la mayor parte de las iniciativas están dirigidas hacia la educación primaria, no abordan un área temática específica y contribuyen al aprendizaje ubicuo con el desarrollo de plataformas tecnológicas. El país que tiene una presencia dominante es Taiwán, los medios de mayor publicación son Computers and Education y Journal of Educational Technology \& Society, y el tipo de artículos que más se publica tiene una orientación hacia la propuesta de aplicaciones prácticas. Los resultados obtenidos sirven de referencia para quienes desde la docencia e investigación desean contribuir con este dominio del conocimiento, y para las autoridades gubernamentales y de instituciones educativas que planeen promover y facilitar el aprendizaje ubicuo.
\end{abstract}

Palabras claves: Aprendizaje en línea; tecnología educacional; enseñanza asistida por ordenador. 
UNICIENCIA Vol. 31, No. 2, pp. 51-67. Julio-diciembre, 2017.

ISSN Electrónico: 2215-3470

URL: www.revistas.una.ac.cr/uniciencia

DOI: http://dx.doi.org/10.15359/ru.31-2.4

Email: revistauniciencia@una.cr

\begin{abstract}
This article analyzes trends in ubiquitous learning research during the period 2000-2015. We searched for articles in English and Spanish that specifically addressed the topics of "ubiquitous learning" or "aprendizaje ubicuo". The selected papers were analyzed in the following categories: target population, subject area, type of contribution, country, publication media and type of paper. The results indicate that most of the initiatives are directed towards primary education; they do not address a specific subject area; and they contribute to the ubiquitous learning domain with the development of technology platforms. The country that has a dominant presence is Taiwan; the more prevalent publication journals are Computers and Education and Journal of Educational Technology \& Society; and the kind of articles that are published have an orientation towards practical applications. The results serve as reference for educators and researchers who wish to contribute to this domain of knowledge, and for government authorities and educational institutions planning to promote and facilitate ubiquitous learning.
\end{abstract}

Keywords: electronic learning; educational technology; computer assisted instruction.

El rápido avance y la popularidad de la comunicación inalámbrica y las tecnologías móviles no solo han alterado la producción de conocimiento, sino también los espacios y tiempos donde el aprendizaje tiene lugar, dando como resultado la emergencia del concepto del aprendizaje ubicuo, también conocido como u-learning.

Liu y Hwang (2010) argumentan que cada vez que se desea aplicar un nuevo tipo de tecnología a los procesos de enseñanza y aprendizaje surgen nuevos paradigmas para orientar el uso, diseño, implementación y evaluación de dichas tecnologías. Sobre la base de esta tendencia, se ha venido experimentado un cambio de paradigma del aprendizaje electrónico convencional (e-learning) al aprendizaje móvil (m-learning) y de este al aprendizaje ubicuo (u-learning).

El aprendizaje ubicuo no selimita a la formación recibida a través del computador o de dispositivos móviles, sino que aprovecha cualquier medio tecnológico que permita recibir información y facilite el aprendizaje. De este modo se deja de visualizar al elearning y mlearning como metodologías aisladas y conceptos separados, para pensar en un contexto ulearning como un aprendizaje que abarca todo lo anterior y, además, incluye todos los medios tecnológicos existentes que puedan ser utilizados para la enseñanza y el aprendizaje (Collazos, Moreno, Yandar, Vicari, \& Coto, 2013).

El aprendizaje ubicuo se basa en la tecnología ubicua. La ubicuidad implica una capacidad especial para la flexibilidad y la adaptación a diferentes contextos. El papel más importante de la tecnología ubicua es la construcción de un entorno de aprendizaje ubicuo, que permita a una persona aprender en cualquier lugar y en cualquier momento (Yahya, Ahmad, \& Jalil, 2010). Esto implica que los dispositivos móviles (tabletas, teléfonos, laptops) tengan un papel importante en el proceso de aprendizaje, sin importar si este se da en el aula o fuera de ella (Hwang, Tsai, \& Yang, 2008).

El aprendizaje ubicuo tiene una serie de características que lo distinguen (Yahya et al., 2010): (1) permanencia: los usuarios y usuarias nunca pierden su información a menos que decidan eliminarla; (2) accesibilidad: la información está siempre disponible cada vez que el estudiantado necesite utilizarla; (3) inmediatez: la información puede ser recuperada inmediatamente por el estudiantado; (4) interactividad: el estudiantado puede interactuar con sus pares, docentes y personal experto con eficiencia y eficacia, a través de diferentes medios de comunicación; y (5) adaptabilidad: el entorno es sensitivo al contexto y se adapta a las necesidades reales estudiantiles para proporcionar la información adecuada. 
La principal ventaja del aprendizaje ubicuo es que traslada el aprendizaje más allá del aula, hacia los ambientes de la vida diaria, apoyado en una tecnología flexible y omnipresente. De esta manera permite estudiantes seleccionar los objetivos de aprendizaje y aplicar su estilo de aprendizaje, a la vez que les permite utilizar cualquier plataforma a su alcance y situarse en casi cualquier parte del mundo. En este nuevo enfoque el personal docente y las instituciones educativas tienen el reto de redefinir la forma en que gestionan el aprendizaje y los procesos educativos. Esto conlleva nuevas exigencias en cuanto a esquemas formativos: a docentes les exige nuevas capacidades y conocimientos, la adaptación permanente al cambio y el paso definitivo del profesorado que enseña al profesorado que facilita el aprendizaje.

En la última década se han realizado muchos estudios referentes al aprendizaje ubicuo (Chen \& Huang, 2012; Hung, Yang, Fang, Hwang, \& Chen, 2014; Kantel, Tovar, \& Serrano, 2010; Ogata, Saito, Paredes, San Martin, \& Yano, 2008; Shih, Chu, \& Hwang, 2011), tanto en las aulas, laboratorios, como en museos y espacios al aire libre. Por ejemplo, en el área de idiomas, un grupo de investigación taiwaneses (Huang, Huang, Huang, \& Lin, 2012) desarrolló el sistema UEVL (ubiquitous English vocabulary learning) que ayuda a estudiantes a experimentar un proceso de aprendizaje sistemático del vocabulario en el que se utiliza la tecnología ubicua para desarrollar el sistema, y videoclips como material de aprendizaje. Chen y $\operatorname{Li}(\underline{2010})$ proponen un sistema ubicuo personalizado y sensible al contexto (PCULS) para el aprendizaje de vocabulario en inglés basado en la ubicación en la que se encuentra el estudiantado, la cual se detecta a través de técnicas de posicionamiento inalámbricos. Liu (2009) desarrolló un ambiente de aprendizaje ubicuo llamado HELLO (Handheld English Language Learning Organization), con sensores y dispositivos de realidad aumentada orientado a mejorar el aprendizaje del lenguaje de los estudiantes.

En el área de las ciencias, hay también múltiples investigaciones y desarrollos, por ejemplo, Hwang, Yang, Tsai, y Yang (2009) desarrollaron un entorno de aprendizaje ubicuo sensible al contexto para guiar a personal de investigación sin experiencia en la práctica de operaciones de difracción de rayos X de un solo cristal. Shih, Chu, Hwang y Kinshuk (2011) adoptaron conceptos del aprendizaje ubicuo para implementar un método (repertory-grid) como un sistema experto para guiar el aprendizaje estudiantil. Por su parte, Hwang, Chu, Shih, Huang y Tsai (2010) proponen un enfoque de árbol orientado a la toma de decisiones para la realización de actividades de observación en las ciencias naturales, con el objetivo último de mejorar la capacidad de estudiantes para clasificar y observar los objetos del mundo real.

Así, ante el surgimiento y auge de este nuevo paradigma, se considera importante conocer las tendencias de investigación actuales en esta área, de tal forma que se tenga un panorama de los avances logrados en la última década. Además, esta información puede ser útil para que quienes investigan en campos relacionados elijan temas que requieren una mayor exploración; para que personas interesadas en el área del aprendizaje ubicuo conozcan cuáles son las revistas con mayor cantidad de publicaciones en la temática, o para facilitar, a los entes responsables de elaborar las políticas educativas, una referencia para la toma de decisiones críticas.

En este contexto, este estudio trata de identificar las tendencias de investigación sobre el aprendizaje ubicuo en el período del 2000 al 2015, a partir de las siguientes preguntas guía: 
UNICIENCIA Vol. 31, No. 2, pp. 51-67. Julio-diciembre, 2017.

URL: www.revistas.una.ac.cr/uniciencia

ISSN Electrónico: 2215-3470

Email: revistauniciencia@una.cr

- ¿Hacia qué tipo de población van dirigidas las iniciativas en este paradigma?

- ¿En qué áreas temáticas se desarrollan estas investigaciones?

- ¿Qué tipos de contribuciones se han realizado para apoyar este tipo de aprendizaje?

- ¿Qué países tienen una mayor presencia en esta área de investigación?

- ¿Qué medios académicos están publicando artículos en este tema, y qué tipo de artículos publican?

- ¿Hacia dónde se dirige el trabajo futuro en el aprendizaje ubicuo?

\section{Trabajos relacionados}

Los estudios para conocer las tendencias de investigación en un área del conocimiento específico no son nuevos. Particularmente en el caso del aprendizaje apoyado con tecnología se han realizado varios en los últimos años. A continuación, una breve reseña de cuatro de ellos:

Hsu y otros (2012) realizaron un análisis de contenido de los artículos en el área del aprendizaje basado en tecnología (technology-based learning -TBL) que fueron publicados del 2000 al 2009 en cinco revistas de investigación ampliamente reconocidas por sus factores de alto impacto (British Journal of Educational Technology (BJET), Computers and Education (C\&E), Educational Technology Research \& Development (ETR\&D), Educational Technology \& Society (ET\&S), and the Journal of Computer Assisted Learning (JCAL)). Los investigadores estaban interesados en conocer, entre otros, ¿cuáles fueron los temas de investigación relacionados al TBL que se publicaron en esos años?; ¿a qué grupos meta iban dirigidos?; y ¿qué dominios del aprendizaje fueron atendidos? Además, querían determinar para cada una de estas preguntas, si existía una diferencia entre el período 2000-2004 y el período 2005-2009.

Hwang y Tsai (2011) realizaron un estudio para conocer el estado de las investigaciones en el tema del aprendizaje móvil y ubicuo del 2001 al 2010. Para esto seleccionaron las mismas

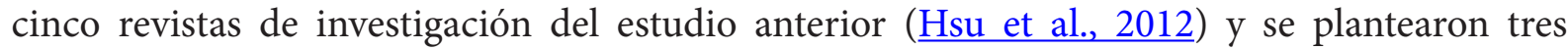
preguntas de investigación: (1) ¿Cuál es el estado de los artículos de aprendizaje móvil y ubicuo publicados del 2001-2010 en las revistas seleccionadas? ¿Está el número de artículos referentes a este tema aumentando o disminuyendo?; (2) ¿Qué grupos meta fueron seleccionados en estas investigaciones?; y (3) Qué dominios de aprendizaje relacionados con el aprendizaje basado en la tecnología fueron adoptadas en estos artículos? ¿Hay algún cambio de dominio entre los primeros 5 años (2001-05) y los segundos 5 años (2006-10)? Para dar respuesta a las interrogantes, trabajaron con 154 artículos finalmente seleccionados.

Baran (2014) realizó un estudio sobre las tendencias de investigación en el uso del aprendizaje móvil para el desarrollo profesional docente. Primeramente realizó una búsqueda en las bases de datos ERIC y Education Research Complete y, posteriormente, amplió la búsqueda a las siguientes revistas de investigación: Journal of Educational Technology and Society, Computers and Education, British Journal of Educational Technology, Journal of Computer Assisted Learning, Educational Technology Research and Development, Journal of Computing in Teacher Education, Journal of Technology and Teacher Education, Journal of Research on Technology in Education, Journal of Digital Learning in Teacher Education. Finalmente sistematizó y codificó 37 artículos en términos de meta-categorías: contexto de formación del profesorado, país, 
tema, tipo, definición de aprendizaje móvil, fiabilidad, validez y confiabilidad, tecnología móvil utilizada, el enfoque pedagógico y los resultados en términos de conocimiento y la práctica de docentes.

Wu y otro (2012) realizaron un análisis de 154 artículos publicados entre el 2003 y el 2010 sobre investigaciones en el área del aprendizaje móvil para dar respuesta a las siguientes preguntas: (1) ¿Cuáles son las principales preguntas de investigación, metodologías y resultados de las investigaciones en torno al aprendizaje móvil?; (2) ¿Qué tipo de dispositivos móviles se utilizan más y cuáles son los tipos de "aprendices móviles"?; (3) ¿Cómo están las diferentes disciplinas y cursos representados en las investigaciones de aprendizaje móvil?; y (4) ¿Cuáles son los artículos altamente citados en estas investigaciones? Los artículos seleccionados para el estudio fueron localizados a través de una búsqueda en bases de datos como: ERIC, Science Direct Onsite (SDOS), SAGE Journal Online, ProQuest, Wiley Inter-Science, ACM Digital Library, JSTOR, Elsevier Science (Elsevier)/SDOL, e informaworld, y de una búsqueda manual en las revistas de investigación: Journal of Computer Assisted Learning, Computer in Human Behavior, British Journal of Educational Technology, Journal of Educational Technology \& Society, y The International Review of Research in Open and Distance Learning.

Los anteriores estudios orientaron, en cierta medida, el que se presenta en este artículo, principalmente en términos de las categorías utilizadas para el análisis de las investigaciones. Sin embargo, como se explica en la siguiente sección, existen importantes diferencias en la forma de buscar y seleccionar los artículos, en las preguntas de investigación formuladas y en cuanto al especial interés de esta investigación de incluir artículos en el idioma español. Dos de los estudios anteriores (Hsu et al., 2012; Hwang \& Tsai, 2011) limitan la búsqueda a cinco revistas específicas, los otros dos estudios (Baran, 2014; Wu et al., 2012) amplían la búsqueda en bases de datos, pero sin incluir artículos publicados en español. De los estudios anteriores, el único que aborda las tendencias de investigación específicamente en el aprendizaje ubicuo es el realizado por Hwang y Tsai (2011); sin embargo, el estudio también incluye iniciativas relacionadas con el aprendizaje móvil (que no necesariamente abordan explícitamente el concepto del aprendizaje ubicuo), está limitado al período 2000-2010 y a las publicaciones realizadas en cinco revistas de investigación, por lo que los resultados no son completamente comparables con los que arroja este artículo.

\section{Metodología}

El primer paso para seleccionar los artículos a estudiar fue realizar una búsqueda del término "ubiquitous learning" en las bases de datos del Sistema de Información Documental (SIDUNA) de la Universidad Nacional (UNA) con dos filtros iniciales: que el año de publicación estuviera comprendido del 2000 hasta el 2015, y que fuera una publicación en revistas arbitradas. Esta búsqueda inicial arrojó un resultado de 1,618 recursos. Posteriormente se aplicó otro filtro para excluir las reseñas de libros, editoriales, libros, actas de conferencias para considerar únicamente los recursos de tipo "artículo". Además, se refinó la selección identificando aquellos medios de publicación que están más relacionados y tienen un mayor impacto en el área de tecnología y aprendizaje (ver Tabla 1).

El último filtro aplicado reduce el número de artículos a 343, de los cuales, por razones prácticas de tiempo y recursos, se seleccionan los 80 primeros, considerados por el sistema de búsquedas como los más relevantes. Dado que los 80 artículos estaban escritos en inglés, y

Mayela Coto-Chotto, Carmen Cordero-Esquivel y Sonia Mora-Rivera

Artículo protegido por licencia Creative Commons: BY-NC-ND / Protected by Creative Commons: BY-NC-ND

Uniciencia es una revista de acceso abierto/ Uniciencia is an Open Access Journal. 
UNICIENCIA Vol. 31, No. 2, pp. 51-67. Julio-diciembre, 2017.

con el fin de ampliar el análisis a artículos en idioma español, se realizó una nueva búsqueda utilizando GOOGLE ACADÉMICO con el término “aprendizaje ubicuo". De igual manera, en este caso se revisan los 80 más relevantes, excluyendo reseñas de libros, editoriales, libros, actas de conferencias para considerar únicamente los recursos de tipo "artículo".

Tabla 1

Medios de publicación que están más relacionados y tienen un mayor impacto en el área de tecnología y aprendizaje

\begin{tabular}{ll} 
Refinar la búsqueda \\
Incluir Excluir $\quad$ Título de la publicación periódica \\
\hline$\square$
\end{tabular}

Nota: Fuente propia de la investigación. Base de datos SIDUNA.

Una primera lectura de los artículos seleccionados determina que hay 50 artículos en inglés y 12 en español que están relacionados directamente y expresamente abordan los tópicos de "ubiquitous learning" y "aprendizaje ubicuo", respectivamente. Los artículos que se descartan se debe a: (1) tratan el término de ubicuidad, pero no en relación con los procesos de enseñanza y aprendizaje, (2) estudian otras modalidades de aprendizaje relacionadas (móvil, e-learning), pero no abordan expresamente el dominio del aprendizaje ubicuo; (3) su contenido no muestra relación con los términos de búsqueda. Los filtros anteriores permiten mantener el estudio dentro del foco previamente definido. 
ISSN Electrónico: 2215-3470

DOI: http://dx.doi.org/10.15359/ru.31-2.4
UNICIENCIA Vol. 31, No. 2, pp. 51-67. Julio-diciembre, 2017.

URL: www.revistas.una.ac.cr/uniciencia

Email: revistauniciencia@una.cr

Los 62 artículos se analizan de acuerdo con un conjunto de categorías (ver Tabla 2) que permiten dar respuesta a las preguntas de investigación planteadas.

Tabla 2

Categorías de análisis

1. Medio de publicación

2. Año de publicación

3. Autores - Nacionalidad

4. Tipo de artículo

- Teórico

- Aplicación práctica

- Mixto

\begin{tabular}{|c|c|}
\hline 5. Área temática & $\begin{array}{l}\text { - Matemáticas } \\
\text { - } \text { Lenguaje/idiomas } \\
\text { - } \text { Arte } \\
\text { - Estudios (bociología, química, física) } \\
\text { - } \text { Ingeniería (computación) } \\
\text { - Salud } \\
\text { - } \text { General } \\
\text { - } \text { Otro definido }\end{array}$ \\
\hline 6. Población meta & $\begin{array}{l}\text { - } \text { Primaria } \\
\text { - Secundaria } \\
\text { - Universidad } \\
\text { - } \text { Adultos mayores } \\
\text { - } \text { Profesores } \\
\text { - } \text { Industria } \\
\text { - } \text { Neneral } \\
\text { - } \text { Otros }\end{array}$ \\
\hline 7. Tipo de desarrollo propuesto & $\begin{array}{l}\text { - Dispositivos (hardware) } \\
\text { - Plataformas (tecnología) } \\
\text { - Software/aplicaciones } \\
\text { - } \text { Infraestructura (redes) } \\
\text { - } \text { Diseño instruccional/enfoque pedagógico } \\
\text { No aplica }\end{array}$ \\
\hline
\end{tabular}

Nota: Fuente propia de la investigación. 
UNICIENCIA Vol. 31, No. 2, pp. 51-67. Julio-diciembre, 2017.

Dichas categorías son comunes en estudios relacionados con tendencias en el área de aprendizaje apoyado por tecnología, por ejemplo (Hsu et al., 2012; Hwang \& Tsai, 2011; Wu et al., 2012).

La categoría “Tipo de artículo", indica el propósito de la investigación presentada, es decir, si el abordaje que se realiza es conceptual, propone una aplicación práctica o es una combinación de ambos. En la Tabla 3, se muestra un ejemplo para cada una de estas.

Tabla 3

Ejemplo de la categoría "Tipo de artículo"

\begin{tabular}{l|l|l}
\hline \multicolumn{1}{c|}{ Artículo } & \multicolumn{1}{c|}{ Descripción } & Categoría \\
\hline $\begin{array}{l}\text { Criteria, Strategies and Research Issues } \\
\text { of Context-Aware Ubiquitous Learning } \\
\text { (Hwang et al., 2008) }\end{array}$ & $\begin{array}{l}\text { Define los criterios básicos de un entorno de } \\
\text { aprendizaje ubicuo y propone varios modelos para } \\
\text { realizar actividades de aprendizaje ubicuas. }\end{array}$ & Teórico \\
\hline $\begin{array}{l}\text { Outdoor Natural Science Learning with an } \\
\text { LFID-Supported Immersive Ubiquitous } \\
\text { Chu, 2009) }\end{array}$ & $\begin{array}{l}\text { Propone un ambiente de aprendizaje ubicuo } \\
\text { con recursos educativos (EULER) basados en la } \\
\text { identificación por radiofrecuencia (RFID), realidad } \\
\text { aumentada (AR), internet, la computación ubicua, } \\
\text { sistemas integrados y tecnologías de bases de datos. }\end{array}$ & Aplicán \\
\hline $\begin{array}{l}\text { A knowledge engineering approach to } \\
\text { developing mindtools for context-aware } \\
\text { ubiquitous learning (Chu, Hwang, \& Tsai, } \\
\text { 2010) }\end{array}$ & $\begin{array}{l}\text { Propone un enfoque de ingeniería del } \\
\text { conocimiento para desarrollar una herramienta } \\
\text { "mindtool" para un contexto de aprendizaje } \\
\text { ubicuo. La herramienta desarrollada se utilizó } \\
\text { en una actividad de aprendizaje de un curso de } \\
\text { ciencias naturales en una escuela primaria. }\end{array}$ & Mixto \\
\hline
\end{tabular}

Nota: Fuente propia de la investigación.

Adicionalmente, en la categoría “Tipo de desarrollo propuesto", los artículos se categorizan en desarrollo de dispositivos físicos o hardware, plataformas tecnológicas (configuraciones de grupos de dispositivos), desarrollos de aplicaciones o software, configuraciones de redes o infraestructura, y desarrollos orientados más hacia la parte pedagógica o de diseño instruccional.

Las restantes categorías están relacionadas con la población a la que va dirigida la investigación ("Población meta") y el área temática o del conocimiento en el que se enfoca la misma (“Área temática”). 
ISSN Electrónico: 2215-3470

DOI: http://dx.doi.org/10.15359/ru.31-2.4
UNICIENCIA Vol. 31, No. 2, pp. 51-67. Julio-diciembre, 2017.

URL: www.revistas.una.ac.cr/uniciencia Email: revistauniciencia@una.cr

\section{Resultados}

De los 62 estudios analizados sobre el aprendizaje ubicuo en el período 2000-2015, puede verse, en la Figura 1, que la frecuencia de publicación tuvo una tendencia creciente del 2008 al 2012, para luego iniciar un descenso. Esta tendencia creciente también se identifica en el estudio realizado por Hwang y Tsai (2011).

\section{NÚMERO DE ARTÍCULOS 2000-2015}

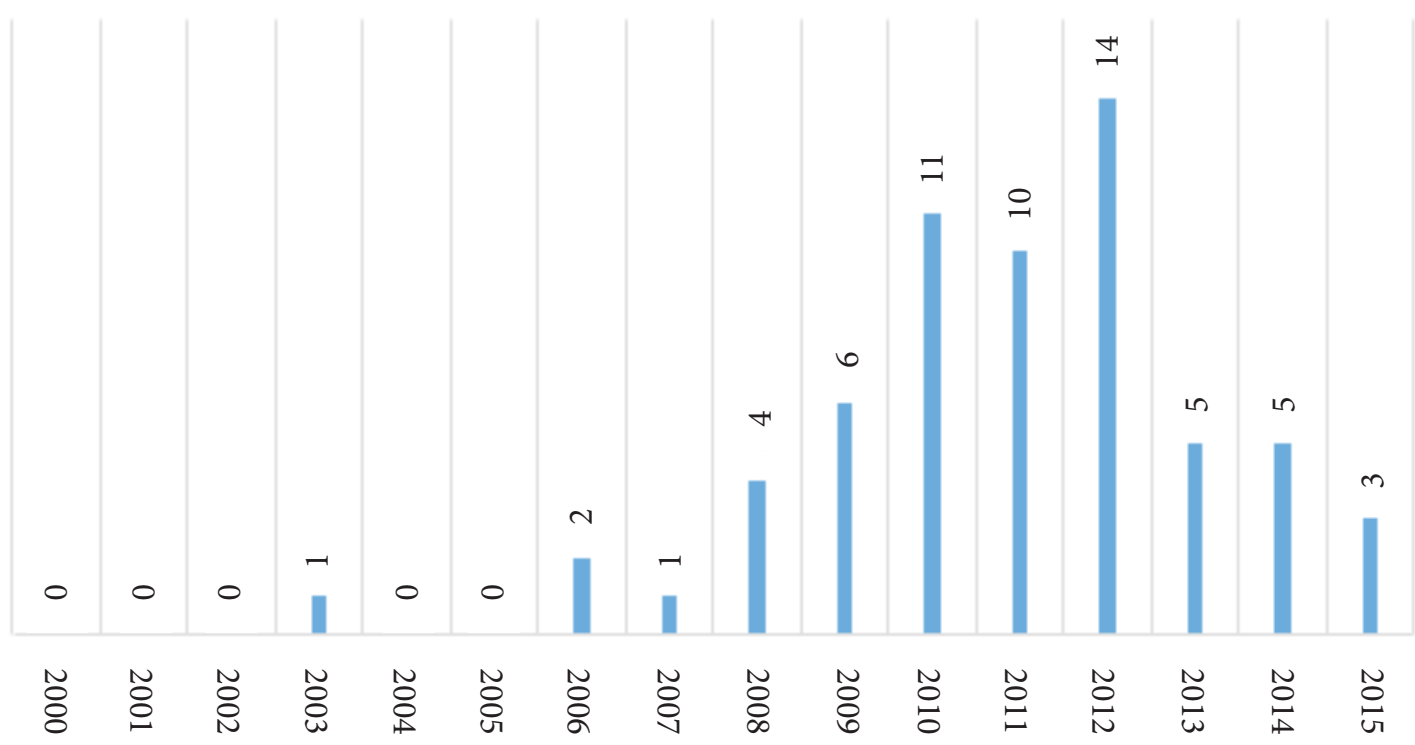

Figura 1. Número de artículos publicados por año entre el 2000 y el 2015

Fuente propia de la investigación.

A continuación, se detallan los resultados del análisis basado en las preguntas de investigación.

\section{Población meta}

En la Figura 2 se aprecia que el nivel de educación primaria ha sido objeto del mayor número (31\%) de las iniciativas de investigación, seguido cercanamente por la educación universitaria (26\%). Es interesante notar que, por el contrario, las iniciativas dirigidas hacia la población secundaria es uno de los más bajos. No se identificó ningún artículo relacionado con poblaciones de adultos mayores o la industria. 
UNICIENCIA Vol. 31, No. 2, pp. 51-67. Julio-diciembre, 2017.

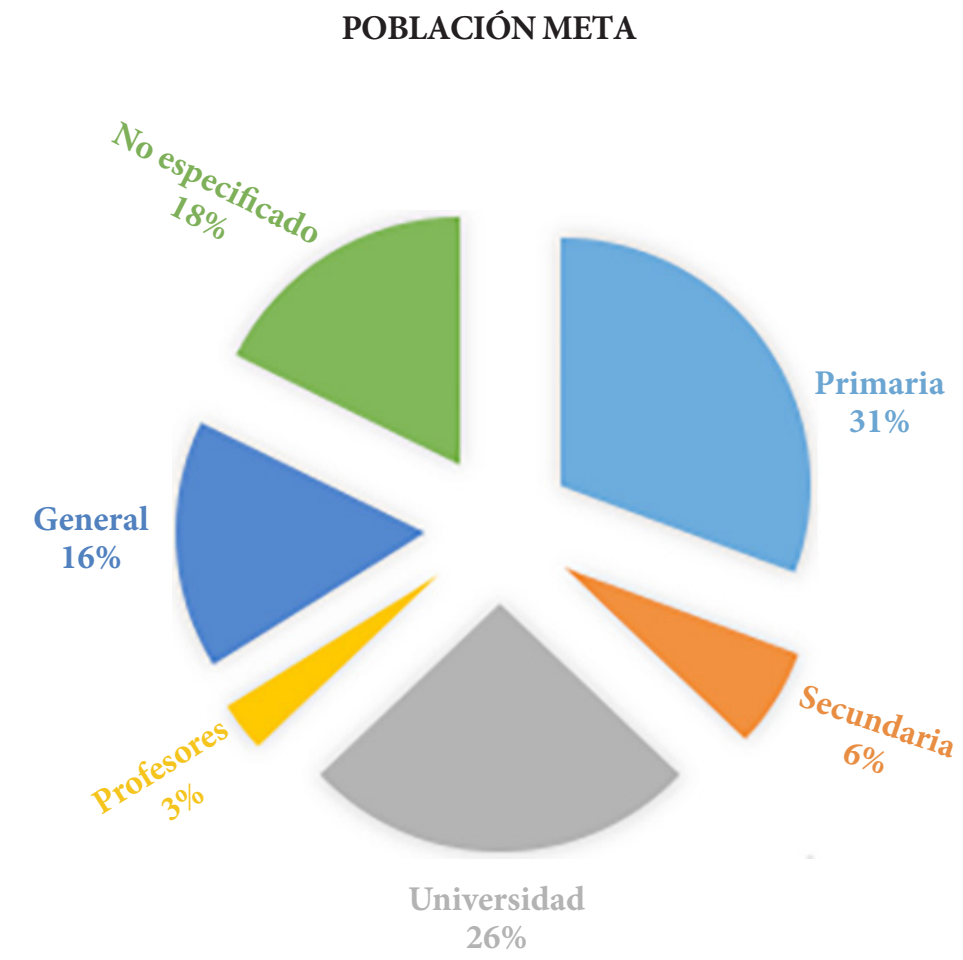

Figura 2. Distribución de los artículos según la población meta a que se dirigen Fuente propia de la investigación.

\section{Áreas temáticas}

La Tabla 4 muestra que una importante cantidad (52\%) de las investigaciones se han realizado en forma independiente del área de aplicación, es decir, son propuestas conceptuales o de aplicación práctica de carácter general ${ }_{2}$ por ejemplo, en esta categoría se encuentra el artículo "La información digital actual, un nuevo modelo de contenido educativo para un entorno de aprendizaje ubicuo" (Rodrigo Alonso \& de Castro Lozano, 2013), que describe una nueva herramienta para la normalización de procesos que busca que el desarrollo de contenidos cumpla con grados de accesibilidad, usabilidad y adaptabilidad aceptables para la mayor gama de entornos y plataformas de aprendizaje que sean posibles. Dicha plataforma puede utilizarse para el desarrollo de contenidos en cualquier área. La segunda área en importancia es la relacionada con las ciencias naturales (21\%), mientras que las áreas de salud y matemáticas son las menos abordadas. 
ISSN Electrónico: 2215-3470

DOI: http://dx.doi.org/10.15359/ru.31-2.4
UNICIENCIA Vol. 31, No. 2, pp. 51-67. Julio-diciembre, 2017. URL: www.revistas.una.ac.cr/uniciencia Email: revistauniciencia@una.cr

Tabla 4

Número y porcentaje de artículos por área temática

\begin{tabular}{lcc}
\hline Área & Número de artículos & Valor porcentual \\
\hline General & 32 & $52 \%$ \\
\hline Ciencias (biología, química, física) & 13 & $21 \%$ \\
\hline Lenguaje/idiomas & 7 & $11 \%$ \\
\hline Arte & 4 & $6 \%$ \\
\hline Ingeniería (computación) & 4 & $6 \%$ \\
\hline Matemáticas & 1 & $2 \%$ \\
\hline Salud & 1 & $2 \%$ \\
\hline & 62 & $100 \%$ \\
\hline
\end{tabular}

Nota: Fuente propia de la investigación.

\section{Tipos de contribución}

En relación con los tipos de desarrollo en el área del aprendizaje ubicuo que han tenido lugar en los últimos 15 años, puede verse en la Tabla 5, que un 47\% de las iniciativas de investigación se orientan al desarrollo de plataformas tecnológicas.

Tabla 5

Número y porcentaje de artículos según tipo de desarrollo

\begin{tabular}{lcc}
\hline Tipos de desarrollo & Número de artículos & Valor porcentual \\
\hline Plataformas (tecnología) & 29 & $47 \%$ \\
\hline No aplica & 14 & $23 \%$ \\
\hline Software/aplicaciones & 10 & $16 \%$ \\
\hline Diseño instruccional/enfoque pedagógico & 7 & $11 \%$ \\
\hline Infraestructura (redes) & 2 & $3 \%$ \\
\hline
\end{tabular}

Nota: Fuente propia de la investigación.

Un ejemplo de un artículo en esta categoría es "Open corpus architecture for personalised ubiquitous e-learning” (Muntean \& Muntean, 2009), el cual propone una novedosa plataforma (múltiples dispositivos, nodos interconectados) de e-learning llamada PEACOCK que proporciona soporte para la mejor selección de contenido y entrega remota de contenido multimedia personalizado a estudiantes.

Del análisis de los artículos puede verse que el grueso de los desarrollos está orientado hacia la parte más tecnológica y en un grado mucho menor hacia el componente pedagógico. 
UNICIENCIA Vol. 31, No. 2, pp. 51-67. Julio-diciembre, 2017.

\section{Medios de difusión y tipos de artículos}

El auge del tema del aprendizaje ubicuo ha provocado que una cantidad de medios de difusión académica publiquen investigaciones relacionadas con este tema. En la Tabla 6, se muestran los medios con representación en la muestra de artículos analizada, donde puede apreciarse un claro dominio de dos de ellos: "Computers and Education" y "Journal of Educational Technology \& Society". Ambos medios son ampliamente reconocidos y cuentan con factores de alto impacto, como lo acredita el Journal Citation Reports del Institute for Scientific Information (ISI). Es importante notar que el único medio en español con cierta representación es la Revista de Educación a Distancia (RED).

Tabla 6

Número de artículos por medio de difusión

\begin{tabular}{|c|c|c|}
\hline Medio de difusión & Número de artículos & Valor porcentual \\
\hline Computers and Education & 20 & $32 \%$ \\
\hline Journal of Educational Technology \& Society & 13 & $21 \%$ \\
\hline British Journal of Educational Technology & 6 & $10 \%$ \\
\hline Journal of Computer Assisted Learning & 6 & $10 \%$ \\
\hline RED - Revista de Educación a Distancia & 5 & $8 \%$ \\
\hline Personal and ubiquitous computing & 3 & $5 \%$ \\
\hline Interactive Learning Environments & 2 & $3 \%$ \\
\hline EKS - Education Knowledge Society & 1 & $2 \%$ \\
\hline IEEE-RITA & 1 & $2 \%$ \\
\hline Revista Electrónica de Investigación y Docencia (REID) & 1 & $2 \%$ \\
\hline Revista Electrónica de Tecnología Educativa & 1 & $2 \%$ \\
\hline Revista Entramados & 1 & $2 \%$ \\
\hline Revista Iberoamericana de Informática Educativa & 1 & $2 \%$ \\
\hline \multirow[t]{2}{*}{ Revista Iberoamericana de Tecnologías del Aprendizaje } & 1 & $2 \%$ \\
\hline & 62 & $100 \%$ \\
\hline
\end{tabular}

Nota: Fuente propia de la investigación.

El tipo de artículo más representativo en la muestra estudiada (Figura 3) son los artículos con una orientación a aplicaciones prácticas (50\%). Como se mencionó anteriormente, este tipo de artículo se enfoca en proponer una solución de aprendizaje ubicuo al contexto analizado, usualmente en la forma de una plataforma tecnológica o una solución de software. 
ISSN Electrónico: 2215-3470 DOI: http://dx.doi.org/10.15359/ru.31-2.4
UNICIENCIA Vol. 31, No. 2, pp. 51-67. Julio-diciembre, 2017.

URL: www.revistas.una.ac.cr/uniciencia Email: revistauniciencia@una.cr

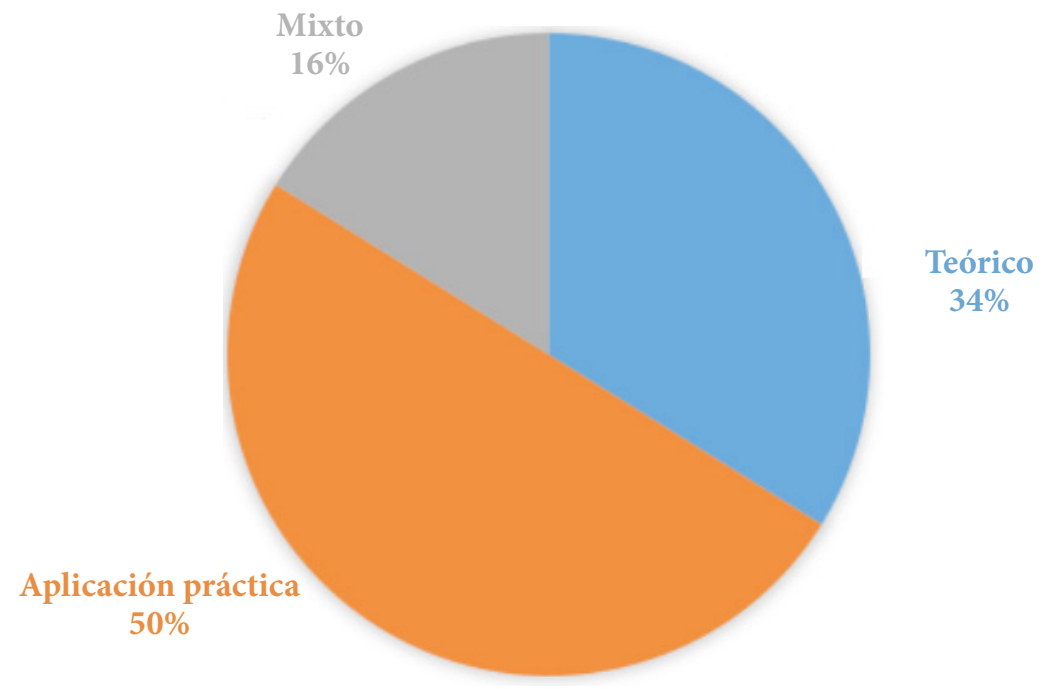

Figura 3. Distribución relativa de artículos según tipo.

Datos propios de la investigación.

\section{Países con mayor representación}

Finalmente, uno de los intereses de este estudio fue identificar el cuerpo académico que está trabajando activamente en esta área. Al analizar los estudios es claro el predominio que actualmente tiene Taiwán (49\%), lo cual puede explicarse en el hecho de que el país cuenta con un plan nacional a partir del 2007 que busca fortalecer el aprendizaje móvil y ubicuo. Además, puede verse de la Figura 4 que España domina casi por completo el ámbito de las publicaciones en el idioma español.

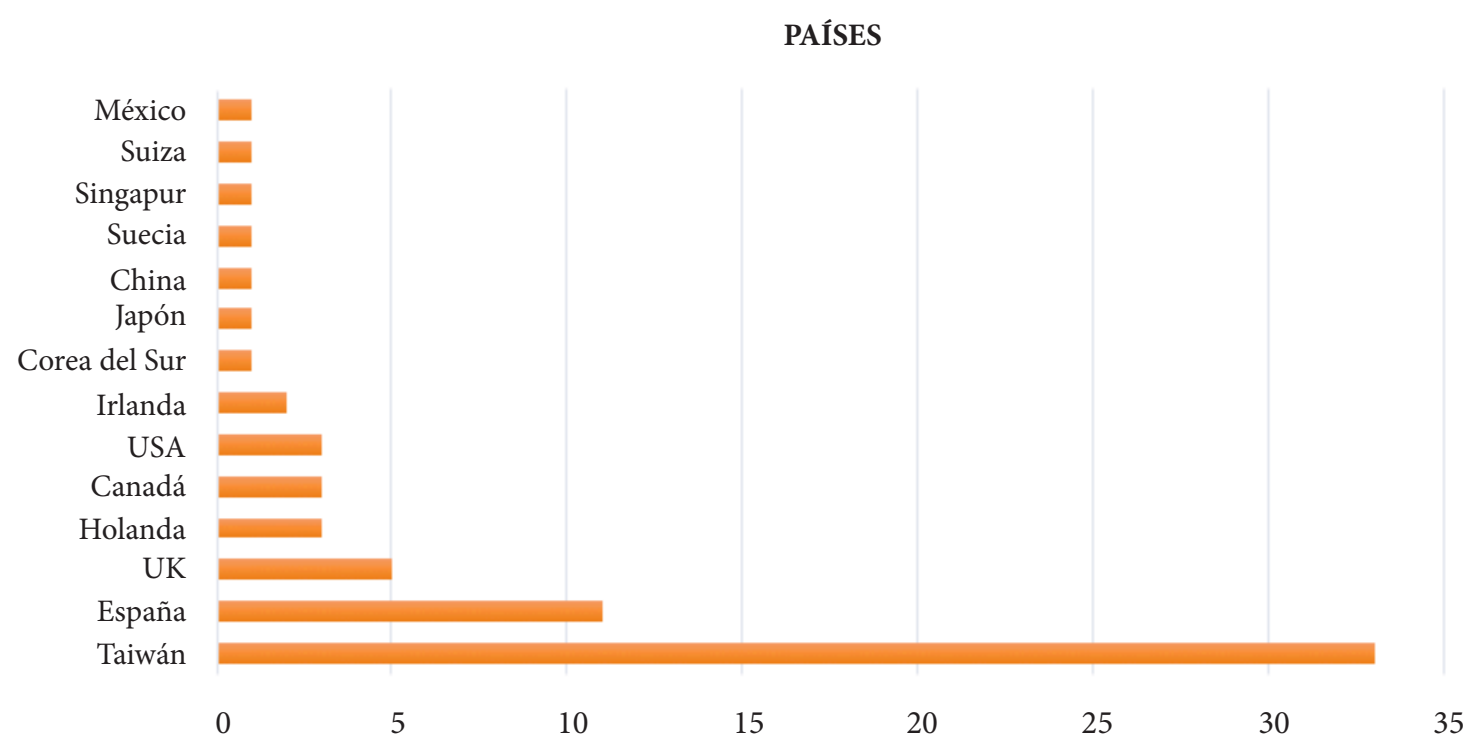

Figura 4. Cantidad de artículos por país de origen.

Datos propios de la investigación. 
UNICIENCIA Vol. 31, No. 2, pp. 51-67. Julio-diciembre, 2017.

ISSN Electrónico: 2215-3470

URL: www.revistas.una.ac.cr/uniciencia

DOI: http://dx.doi.org/10.15359/ru.31-2.4

Email: revistauniciencia@una.cr

\section{Conclusiones}

Los anteriores resultados pretenden dar una muestra de las tendencias de investigación actuales en el aprendizaje ubicuo. Al analizar estos resultados debe tenerse en mente que el estudio realizado no pretende ser exhaustivo, las conclusiones que se obtienen parten del estudio de 62 artículos, por lo que claramente solo muestran una tendencia. Sin embargo, a pesar de contar con una muestra relativamente pequeña, los resultados obtenidos están alineados con otras investigaciones similares (

¿Hacia qué tipo de población van dirigidas las iniciativas en este paradigma? Los datos muestran que las poblaciones meta que son mayormente seleccionadas para las iniciativas relacionadas con el aprendizaje ubicuo son el nivel de primaria en un $31 \%$ y el nivel universitario en un $26 \%$, muy por detrás queda el nivel de secundaria (6\%) y el sector docente (3\%).

¿En qué áreas temáticas se desarrollan estas investigaciones? La gran mayoría de las investigaciones (39\%) tienen un ámbito de aplicación general, es decir, sin un área disciplinaria de aplicación específica. En el área de ciencias, que comprende biología, química y física, también se han generado una buena cantidad de investigaciones (21\%), y las áreas de la salud y matemáticas son las menos representadas ( $2 \%$ cada una).

¿Qué tipos de contribuciones se han realizado para apoyar este tipo de aprendizaje? Prácticamente la mitad de los artículos revisados (47\%) se enfocan en el desarrollo de plataformas tecnológicas para apoyar el aprendizaje ubicuo. Este tipo de plataforma generalmente involucra múltiples dispositivos móviles, sensores, redes de comunicación, dispositivos de realidad aumentada, entre otras. Un $16 \%$ propone el desarrollo de software o aplicaciones móviles y únicamente un $11 \%$ analiza los aspectos de diseño instruccional o enfoques pedagógicos que deben acompañar al nuevo paradigma de aprendizaje.

¿Qué países tienen una mayor presencia en esta área de investigación? Claramente Taiwán es el país con mayor presencia en publicaciones (49\%), lo cual está asociado, como se comentó anteriormente, a la segunda fase de un plan nacional para promover en las instituciones educativas el aprendizaje móvil y ubicuo. En el ámbito de las publicaciones en inglés, Taiwán es seguido por el Reino Unido (7\%), pero con una brecha significativa entre ambos países. Cabe recalcar que en el estudio realizado por Hwang y Tsai (2011) se evidencia también este predominio de Taiwan en el período 2005-2010. En el ámbito de las publicaciones en idioma español, únicamente España y México tienen representación, pero con un claro dominio de la primera.

¿Qué medios académicos están publicando artículos en este tema, y qué tipo de artículos publican? Las revistas de investigación que publican más artículos relacionados con el aprendizaje ubicuo son: Computers and Education $(\mathrm{C} \& \mathrm{E})$ con un $32 \%$ de las publicaciones, Journal of Educational Technology \& Society (ET\&S) con un 21\% y British Journal of Educational Technology (BJET) y Journal of Computer Assisted Learning (JCAL) con un 10\% cada una. Cabe mencionar que estas cuatro revistas figuran entre las seleccionadas por otros estudios similares (Baran, 2014; Hsu et al., 2012; Hwang \& Tsai, 2011; Wu et al., 2012). El idioma dominante de las publicaciones es el inglés (82\%). No se identificaron publicaciones en español en las bases de datos de mayor alcance por lo que se necesitó ampliar la búsqueda por medio de Google Académico, dando como resultado que la única revista en español que tiene cierta presencia en este ámbito del conocimiento es la Revista de Educación a Distancia (RED).

Con respecto al tipo de artículo que se publica, el 50\% son de aplicación práctica (aplicaciones 
móviles, plataformas tecnológicas), el 34\% son teóricos o conceptuales y el $16 \%$ son mixtos, en el sentido de que desarrollan temas que aportan elementos conceptuales al dominio del aprendizaje ubicuo y lo acompañan con alguna solución práctica.

¿Hacia dónde se dirige el trabajo futuro en el aprendizaje ubicuo? Una parte considerable de los artículos analizados indica la importancia de considerar el contexto en el que el aprendizaje toma lugar e incorporarlo a la experiencia de aprendizaje (context-aware u-learning), además de diseñar entornos de aprendizaje centrados en los hábitos y preferencias de cada individuo (personalización).

La ubicuidad permite que las experiencias de aprendizaje trasciendan el salón de clases, por lo que muchos de los artículos promueven el diseño de experiencias que integran aprendizajes de tipo formal, informal, situado y experiencial. En general, el llamado al personal docente e investigativo es a promover el aprendizaje como un proceso más continuo, integrado al flujo de las actividades humanas y, por lo tanto, más situado, colaborativo y contextual.

En este trabajo se ha revisado el avance de la investigación en el aprendizaje ubicuo durante el período 2000-2015. Los resultados obtenidos pueden ser una buena referencia para quienes educan e investigan que desean contribuir a este dominio del conocimiento. Por otra parte, los resultados pueden guiar a las autoridades gubernamentales y de instituciones educativas a asignar recursos y ejecutar proyectos que permitan apoyar futuras investigaciones y desarrollo de aplicaciones que promuevan y facilitan el aprendizaje ubicuo.

Dado que la muestra final utilizada en este estudio es relativamente pequeña y que no se incluyeron las actas de congresos relevantes y otras publicaciones relacionadas con el tema (por ejemplo, las publicaciones de IEEE), se sugiere que una investigación futura amplíe las fuentes de datos tanto para investigaciones en inglés como en español.

\section{Agradecimientos}

Este artículo es desarrollado como parte del proyecto "RED u-CSCL: Red Iberoamericana de apoyo a los procesos de enseñanza-aprendizaje de competencias profesionales a través de entornos ubicuos y colaborativos, código CYTED 513RT0481", y como parte del proyecto de la Universidad Nacional: "Desarrollo e implementación de un modelo pedagógico de apoyo a los procesos de enseñanza-aprendizaje de competencias profesionales a través de entornos ubicuos y colaborativos en el marco de la red U-CSCL", código 0406.

\section{Referencias}

Baran, E. (2014). A Review of Research on Mobile Learning in Teacher Education. Educational Technology \& Society, 17(4), 17-32.

Chen, C., \& Huang, T. (2012). Learning in a u-Museum: Developing a context-aware ubiquitous learning environment. Computers \& Education, 59(3), 873-883. http://doi.org/10.1016/j. compedu.2012.04.003

Chen, C.-M., \& Li, Y.-L. (2010). Personalised context-aware ubiquitous learning system for supporting effective English vocabulary learning. Interactive Learning Environments, 18(4), 341-364. http:// dx.doi.org/10.1080/10494820802602329

Chu, H.-C., Hwang, G.-J., \& Tsai, C.-C. (2010). A Knowledge Engineering Approach to Developing Mindtools for Context-Aware Ubiquitous Learning. Computers \& Education, 54(1), 289-297. http:// doi.org/10.1016/j.compedu.2009.08.023 
UNICIENCIA Vol. 31, No. 2, pp. 51-67. Julio-diciembre, 2017.

ISSN Electrónico: 2215-3470

URL: www.revistas.una.ac.cr/uniciencia

DOI: http://dx.doi.org/10.15359/ru.31-2.4

Email: revistauniciencia@una.cr

Collazos, C., Moreno, A., Yandar, M., Vicari, R., \& Coto, M. (2013). Propuesta metodológica de apoyo a los procesos de enseñanza-aprendizaje a través de entornos ubicuos y colaborativos: u-CSCL. Presentado en VIII Congreso Colombiano de Computación - 8CCC.

Hsu, Y. C., Ho, H. N., Tsai, C., Hwang, G. J., Chu, H. C., Wang, C. Y., \& Chen, N. S. (2012). Research Trends in Technology-based Learning from 2000 to 2009: A content Analysis of Publications in Selected Journals. Educational Technology \& Society, 15(2), 354-370.

Huang, Y.-M., Huang, Y.-M., Huang, S.-H., \& Lin, Y.-T. (2012). A ubiquitous English vocabulary learning system: Evidence of active/passive attitudes vs. usefulness/ease-of-use. Computers \& Education, 58(1), 273 - 282. http://doi.org/http://dx.doi.org/10.1016/j.compedu.2011.08.008

Hung, I., Yang, X., Fang, W., Hwang, G. J., \& Chen, N. (2014). A context-aware video prompt approach to improving students' in-field reflection levels. Computers \& Education, 70, 80-91. http://doi. org/10.1016/j.compedu.2013.08.007

Hwang, G. J., Tsai, C., \& Yang, S. J. (2008). Criteria, strategies and research issues of context-aware ubiquitous learning. Educational Technology \& Society, 11(2), 81-91.

Hwang, G.-J., \& Tsai, C.-C. (2011). Research trends in mobile and ubiquitous learning: a review of publications in selected journals from 2001 to 2010. British Journal of Educational Technology, 42(4), E65-E70. http://doi.org/10.1111/j.1467-8535.2011.01183.x

Hwang, G.-J., Chu, H.-C., Shih, J.-L., Huang, S.-H., \& Tsai, C.-C. (2010). A Decision-Tree-Oriented Guidance Mechanism for Conducting Nature Science Observation Activities in a Context-Aware Ubiquitous Learning Environment. Educational Technology \& Society, 13(2), 53-64.

Hwang, G.-J., Yang, T.-C., Tsai, C.-C., \& Yang, S. J. H. (2009). A context-aware ubiquitous learning environment for conducting complex science experiments. Computers \& Education, 53(2), 402-413. http://doi.org/http://dx.doi.org/10.1016/j.compedu.2009.02.016

Kantel, E., Tovar, G., \& Serrano, A. (2010). Diseño de un entorno colaborativo móvil para apoyo al aprendizaje a través de dispositivos móviles de tercera generación. Revista Iberoamericana de Tecnologías del Aprendizaje, 5(4), 146-151.

Liu, G., \& Hwang, G. J. (2010). A key step to understanding paradigm shifts in e-learning: towards context-aware ubiquitous learning. British Journal of Educational Technology, 41(2). http://doi. org/10.1111/j.1467-8535.2009.00976.x

Liu,T.(2009).Acontext-awareubiquitouslearningenvironmentforlanguagelisteningandspeaking.Journal of Computer Assisted Learning, 25(6), 515-527. http://dx.doi.org/10.1111/j.1365-2729.2009.00329.x

Liu, T.-Y., Tan, T.-H., \& Chu, Y.-L. (2009). Outdoor Natural Science Learning with an RFID-Supported Immersive Ubiquitous Learning Environment. Educational Technology \& Society, 12(4), 161-175.

Muntean, C. H., \& Muntean, G. M. (2009). Open corpus architecture for personalised ubiquitous e-learning. Personal and Ubiquitous Computing, 13(3), 197-205. http://dx.doi.org/10.1007/ $\underline{\text { s00779-007-0189-5 }}$

Ogata, I., Saito, N. A., Paredes, R. G., San Martin, G. A., \& Yano, Y. (2008). Supporting classroom activities with the BSUL environment," in 2005. WMTE 2005. IEEE International Workshop on, vol., no., pp.8 pp.-, 28-30 Nov. 2005 doi: 10.1109/WMTE.2005.61. Journal of Educational Technology \& Society, 11(1), 1-16.

Rodrigo Alonso, M., \& de Castro Lozano, C. (2013). La información digital actual, un nuevo modelo de contenido educativo para un entorno de aprendizaje ubicuo. RED. Revista de Educación a Distancia, 39, 18-34. Recuperado de http://google.redalyc.org/articulo.oa?id=54729539002 
ISSN Electrónico: 2215-3470

DOI: http://dx.doi.org/10.15359/ru.31-2.4
UNICIENCIA Vol. 31, No. 2, pp. 51-67. Julio-diciembre, 2017. URL: www.revistas.una.ac.cr/uniciencia Email: revistauniciencia@una.cr

Shih, J., Chu, H., \& Hwang, G. J. (2011). An investigation of attitudes of students and teachers about participating in a context-aware ubiquitous learning activity. British Journal of Educational Technology, 42(3), 373-394. http://doi.org/10.1111/j.1467-8535.2009.01020.x

Wu, W. H., Wu, Y. C. J., Chen, C. Y., Kao, H. Y., Lin, C. H., \& Huang, S. H. (2012). Review of trends from mobile learning studies: A meta-analysis. Computers \& Education, 59(2), 817-827. http://dx.doi. org/10.1016/j.compedu.2012.03.016

Yahya, S., Ahmad, E. A., \& Jalil, K. A. (2010). The definition and characteristics of ubiquitous learning: A discussion. International Journal of Education and Development using Information and Communication Technology (IJEDICT), 6(1), 117-127

\section{(2) $\odot \Theta \Theta$}

Tendencias de investigación en el aprendizaje ubicuo: un micro estudio de publicaciones seleccionadas del 2000 al 2015 (Mayela Coto-Chotto y otros) por Revista Uniciencia se encuentra bajo una Licencia CreativeCommons AtribuciónNoComercial-SinDerivadas 3.0 Unported. 